\title{
Erratum
}

\section{Non-Empirical LCAO-MO-SCF-CI Calculations on Organic Molecules with Gaussian Type Functions}

I. G. Cstzmadia, M. C. HarRison, J. W. Moskowitz, and B. T. Sutcliffe

Theoret. chim. Acta (Berl.) 6, 191 (1966)

Received January 9, 1967

1. Tab. 5 lists electronic attraction energy values for $\mathrm{Be}_{2}$. In order to obtain the total energy one has to add the nuclear repulsion energy to the values quoted which is 4.23280 Hartree a.u. at the chosen internuclear separation (3.78 Bohr a.u.).

2. The correct Eq. (20) is :

$$
e^{-\alpha r_{A}^{2}} \cdot e^{-\beta r_{B}^{2}}=\mathrm{const} \cdot e^{-(\alpha+\beta) r_{E}^{2}}
$$

This corrected equation holds for two Gaussians. If one multiplies two Gaussian type functions (GTF) as defined by $\mathrm{Eq}$. (18) the product itself is a Gaussian (apart from a finite power series as a factor) with a centre somewhere on the line segment AB.

3. The correct reference number for Koopmans' theorem is [24] on page 198.

4. The elements of the total and open density matrices $\underline{Q}^{t}$ and $\varrho^{o}$ with correct indices are $\varrho_{k l}^{t}$ and $\varrho_{l k l}^{o}$ and they should replace the symbols given on the left hand side of Eqs. (41) and (42) respectively.

The authors are indebted to E. ScHwaRz for calling their attentions to the above errors. 